vessels. In contrast, this lesion was easily identified by the nodule seen macroscopically. The propensity of angiolymphoid hyperplasia with eosinophilia to bleed was seen in this case, highlighting a potentially fatal outcome of an unusual lesion previously unreported at this site.

1 Razquin S. Mayayo E, Citores MA, Alvira R. Angiolymphoid hyperplasia with eosinophilia of the tongue: report of a case and review of the literature. Hum Pathol 1991;22:837-9.

2 Toeg A, Kermish M, Grishkan A, Temkin D. Histiocytoid hemangioma of the oral cavity: a report of two cases. $\mathcal{F}$ Oral Maxillofac Surg 1993;51:812-14.
3 Reed RJ, Terazakis N. Subcutaneous angioblastic lymphoid hyperplasia with eosinophilia (Kimura's disease). Cancer 1972;29:489-97.

4 Olsen TJ, Helwig EB. Angiolymphoid hyperplasia with eosinophilia: a clinicopathologic study of 116 patients. $\mathcal{F}$ Am Acad Dermatol 1985;12:781-96.

5 Fetsch JF, Weiss SW. Observations concerning the pathogenesis of epithelioid hemangioma (angiolymphoid hyperplasia). Modern Pathology 1991;4:449-55.

6 Henry PG, Burnett JW. Angiolymphoid hyperplasia with eosinophilia. Arch Dermatol 1978;114:1168-72.

7 Kung ITM, Gibson JB, Bannatyne PM. Kimura's disease: a clinicopathological study of 21 cases and its distinction from angiolymphoid hyperplasia with eosinophilia. Pathology 1984;16:39-44.

8 Googe PB, Harris NL, Mihim MC. Kimura's disease and angiolymphoid hyperplasia with eosinophils. Two distinct histopathological entities. F Cutan Pathol 1987;14:263-71.

\title{
Mucinous cystadenoma of the appendix with raised serum carcinoembryonic antigen concentration: clinical and pathological features
}

\author{
T Shimizu, M Shimizu, K Kawaguchi, W Yomura, Y Ihara, T Matsumoto
}

Shimizu Women's Clinic, Takarazuka, Japan

T Shimizu

W Yomura

\section{Department of}

Pathology, Kawasaki

Medical School,

Kurashiki, Japan

M Shimizu

Division of

Gastroenterology,

Department of

Medicine

T Matsumoto

Department of

Obstetrics and

Gynecology, Tazuke

Kofukai Medical

Research Institute,

Kitano Hospital,

Osaka, Japan

K Kawaguchi

\section{Department of}

Obstetrics and

Gynecology, Kobe

City General Hospital,

Kobe, Japan

Y Ihara

Correspondence to:

Dr Shimizu, 2-2-4

Minamiguchi, Takarazuka,

Hyogo, Japan 665

Accepted for publication 13 May 1997

\begin{abstract}
A case of mucinous cystadenoma mimicking ovarian cancer is reported. Serum carcinoembryonic antigen (CEA) concentration was raised, and computed tomography of the abdomen and pelvis demonstrated a long oval shaped cystic mass measuring $9 \mathrm{~cm}$ in length on the right anterior side of the uterus. Because of possible right ovarian cancer, laparotomy was performed and the mass was found to be a mucinous cystadenoma of the appendix. This case indicates that mucinous cystadenoma of the appendix may show an unusual presentation including its location as well as the high serum CEA, mimicking ovarian cancer. Therefore, gynaecologists as well as gastroenterologists should consider its possibility as a differential diagnosis of the right adnexal mass in a patient without previous appendectomy.

(F Clin Pathol 1997;50:613-614)
\end{abstract}

Keywords: mucinous cystadenoma; mucocele; appendix; carcinoembryonic antigen; ovarian cancer

An enlarged appendix with luminal dilatation by mucus has generally been called mucocele. ${ }^{1}$ Higa et $a l^{2}$ in 1973, investigated cases of mucocele of the appendix and they classified their lesions into three groups: mucosal hyperplasia, mucinous cystadenoma, and mucinous cystadenocarcinoma. These investigators also recommended avoiding the term mucocele. However, mucocele is still used for gross description, rather than histological diagnosis. ${ }^{1}$ In addition, the term retention mucocele, also called simple mucocele, is still applied to a pathological description of the mucinous dilatation of the appendiceal lumen resulting from any cause other than epithelial proliferation. ${ }^{34}$ On the other hand, when an appendiceal adenoma secretes large amounts of mucus resulting in a clinically palpable cystic lesion, the term cystadenoma of the appendix preferably is used.

We report a case of mucinous cystadenoma of the appendix accompanied by raised serum carcinoembryonic antigen (CEA) concentrations. The case was unique because of the location of the tumour, which led to an initial diagnosis of ovarian cancer.

\section{Case report}

A 75 year old Japanese woman (gravida 6, para 6) underwent a medical check up that identified raised serum CEA. Two months later, she visited a women's clinic and was referred to our hospital with a putative diagnosis of right ovarian cancer. Laboratory data including CA125 and CA19-9 were all within normal limits, except for a raised value for CEA $(17.7 \mathrm{ng} / \mathrm{ml}$; normal range 0-2.5). While there was no mass palpable on the abdomen, physical examination of the pelvis revealed a sausage shaped mass in the right adnexal region measuring $8 \mathrm{~cm}$ in length. There was no lymphadenopathy and cytological examination of the cervix and vagina showed no malignancy. Computed tomography (CT) and ultrasonography of the abdomen and pelvis demonstrated an elliptical cystic mass measuring $9 \mathrm{~cm}$ in length on the right anterior side of the uterus (fig 1). Barium enema examination showed no abnormalities within the colorectum.

Laparotomy was performed because of possible right ovarian cancer. During the operation, no ascites was noted. Operative findings 


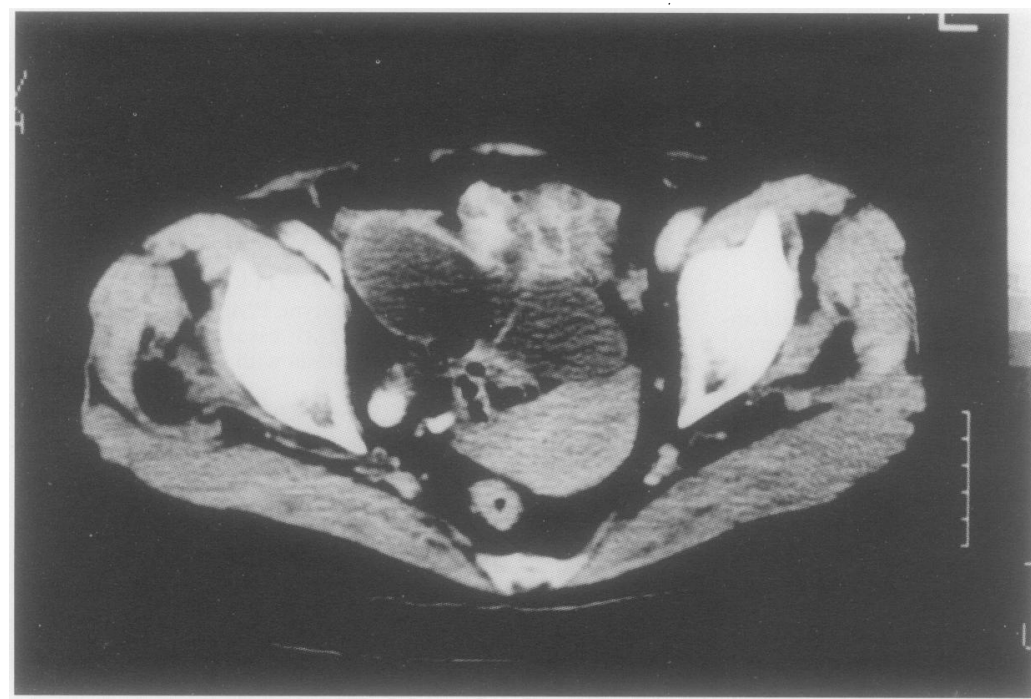

Figure 1 Computed tomography of the abdomen and pelvis showing a well demarcated elliptical cystic mass on the right anterior side of the uterus.

confirmed that the mass was located in the right anterior side of the uterus, and that it originated from the appendix, rather than from the ovary. Because serosal invasion and regional lymph node enlargement were not evident, appendectomy was performed.

Immediately after the operation serum CEA concentrations became normal. The patient has been well for 10 years since the surgery without any symptoms or recurrence.

The resected appendix was diffusely enlarged, measuring $8 \mathrm{~cm}$ in length and $3 \mathrm{~cm}$ in maximal diameter. On sectioning, the lesion was cystic and contained large amounts of mucus. Microscopically, the cyst wall was lined by columnar mucinous epithelium with tall, crowded, and basally located nuclei (fig 2), which was immunohistochemically positive for CEA. There was no evidence of malignant change in $5 \mathrm{~mm}$ thick serial sections from the resected specimens.

\section{Discussion}

Carr et $a P^{6}$ recently classified non-carcinoid epithelial tumours of the appendix into the fol-

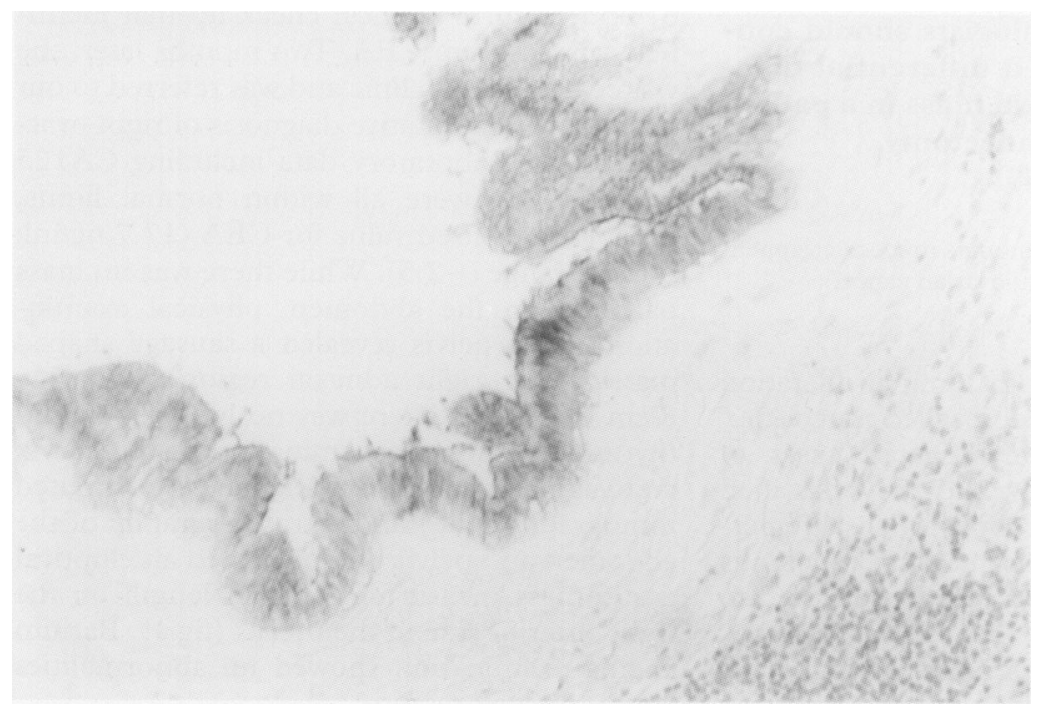

Figure 2 The cyst wall of the tumour lined by mucinous columnar epithelium with tall, crowded, and basally located nuclei. These findings are consistent with mucinous cystadenoma of the appendix. (Haematoxylin and eosin stain;original magnification $\times 100$ ). lowing five groups: simple mucocele, hyperplastic polyp, adenoma, mucinous tumour of undetermined malignant potential, and adenocarcinoma. In that classification, the application of the term mucocele is limited to inflammatory or obstructive lesions showing neither mucosal hyperplasia nor neoplasia. The term mucocele is, however, still confusing because it is frequently applied by clinicians to mucinous tumours of the appendix, ${ }^{78}$ and pathologists use the terms retention mucocele or simple mucocele. ${ }^{356}$ In this report we specified the lesion as mucinous cystadenoma, according to the classification by Carr et al.

The tumour in the present case was characterised by raised serum CEA concentrations and an unusual location. These features were initially suggestive of ovarian cancer. While patients with mucinous cystadenocarcinoma, a rare malignant counterpart of cystadenoma, have been known to manifest high serum CEA values, ${ }^{9}$ there has been no reported case of mucinous cystadenoma of the appendix with raised serum CEA. This rare association seems to be partly explained as serum CEA has not been measured routinely preoperatively in cases of mucinous cystadenoma of the appendix.

A correct preoperative diagnosis of cystic mass of the appendix can readily be made with CT and ultrasound. However, these procedures are not perfect, because there are cases of variable location and appearance ${ }^{78}$ as demonstrated in our case. Such an unusual location of mucocele of the appendix, which was initially diagnosed as twisted ovarian cyst, has been described. ${ }^{10}$

In conclusion, we present an uncommon case of mucinous cystadenoma of the appendix that mimicked ovarian cancer. Our case suggests that appendiceal tumour should be considered as a differential diagnosis for the right adnexal mass of subjects without previous appendectomy. Because serum CEA possibly increases in subjects with the tumour, pathologists should examine the whole resected specimen in this type of tumour to rule out completely the coexisting carcinoma.

1 Schwartz MR. Mucoceles and epithelial tumors of the appendix: a new look at nomenclature and prognostic factors. Adv Anat Pathol 1996;3:355-61.

2 Higa E, Rasai J, Pizzimbona CA, Wise L. Mucosal hyperplasia, mucinous cystadenoma, and mucinous cystadenocarcinoma of the appendix: a muchas appendiceal "mucocele". Cancer 1973;32:1525-41.

3 Fenoglio-Preiser CM, Pascal RR, Perzin KH. Tumors of the intestines. Washington DC: Armed Forces Institute of Pathology, 1990:251-64.

4 Wiliams RA, Whitehead R. Non-carcinoid epithelial tumours of the appendix: a proposed classification. Pathology 1986;18:50-3.

5 Carr NJ, McCarthy WF, Sobin LH. Epithelial noncarcinoid tumors and tumor-like lesions of the appendix. A clinicopathologic study of 184 patients with a multivariate analysis of prognostic factors. Cancer 1995;75:757-68.

6 Carr NJ, Sobin LH. Epithelial noncarcinoid tumors and tumor-like lesions of the appendix [letter]. Cancer 1995;76: 2383-4.

7 Sandler MA, Pearlberg JL, Madrazo BL. Ultrasonic and computed tomographic features of mucocele of the appendix. $\mathcal{F}$ Ultrasound Med 1984;3:97-100.

8 Schwimer SR, Joseph BJ, Lebovic J. Unusual presentation of a mucocele of the appendix. F Ultrasound Med 1987;6:85-7.

Noritake N, Ito Y, Yamakita N, Asuma S, Shimokawa K, Noritake N, Ito Y, Yamakita N, Asuma S, Shimokawa K,
Miura K. A case of primary mucinous cystadenocarcinoma Miura K. A case of primary mucinous cystadenocarcinoma
of the appendix with elevated serum carcinoembryonic of the appendix with elevated serum carcin
antigen (CEA). $\mathcal{F} p n \mathcal{F}$ Med 1990;29:642-6.

10 Zidan FMA, Al-Hilaly MA, AL-Atrabi N. Torsion of a mucocele of the appendix in a pregnant woman. Acta Obstet Gynecol Scand 1992;71:140-2. 